\title{
Zooarqueología de tiempos históricos en el bosque andino de la Patagonia Argentina. \\ Continuidades y cambios en el Valle del Manso Inferior
}

\section{Zooarchaeology of historical times in the Andean forest of Patagonia Argentina. Continuities and changes in the Lower Manso River Valley}

\author{
PABLO MARCELO FERNÁNDEZ ${ }^{1} \&$ MERCEDES GRISEL FERNÁNDEZ² \\ ${ }^{1}$ Instituto Nacional de Antropología y Pensamiento Latinoamericano (INAPL), Consejo Nacional de Investigaciones \\ Científicas y Técnicas (CONICET) / Universidad de Buenos Aires (UBA) \\ email: pablomfernandez69@yahoo.com.ar \\ ${ }^{2}$ Consejo Nacional de Investigaciones Científicas y Técnicas (CONICET) / Instituto Nacional de Antropología \\ y Pensamiento Latinoamericano (INAPL), \\ email: fernandez_mercedesgrisel@hotmail.com
}

(Received 22 May 2018; Revised 25 June 2018; Accepted 10 July 2018)

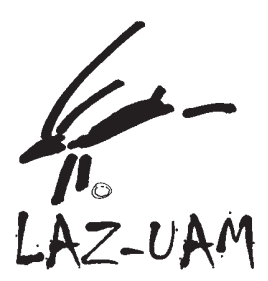

RESUMEN: La introducción y disponibilidad de animales de origen europeo tuvo un impacto variable en la subsistencia de los cazadores-recolectores que habitaron el centro-norte de la Patagonia argentina. De acuerdo con las fuentes documentales, en ciertos sectores del bosque andino la presencia de fauna introducida data del siglo XVII, momento a partir del cual se habrían desencadenado profundas transformaciones en la subsistencia de las poblaciones indígenas que habitaban este ambiente y zonas aledañas. No obstante, estos cambios han sido escasamente estudiados desde una perspectiva zooarqueológica. El análisis de conjuntos óseos datados en tiempos históricos procedentes del valle del río Manso inferior (suroeste de la provincia de Río Negro) revela la existencia de continuidades y cambios en la interacción con los animales. Mientras que el huemul (Hippocamelus bisulcus) mantiene su rol central en la subsistencia, establecido desde el Holoceno temprano, en los tiempos históricos se registra el aporte en muy bajas proporciones de especies complementarias. Entre ellas está el caballo (Equus caballus), cuyos restos sugieren el consumo de carne y médula de un modo similar al registrado entre los ungulados silvestres. Estos resultados muestran la importancia del desarrollo de estudios zooarqueológicos que contemplen una perspectiva comparativa de largo plazo y a escala local.

PALABRAS CLAVE: ZOOARQUEOLOGÍA, TIEMPOS HISTÓRICOS, PATAGONIA, HUEMUL, CABALLO

ABSTRACT: Faunal species introduced from Europe had a variable impact on the subsistence of North-Center Argentine Patagonian hunter-gatherer societies. According to documentary sources, in certain sectors of the Andean forest the presence of introduced fauna dates back to the $17^{\text {th }}$ century. At that time, deep transformations in the subsistence of the indigenous populations that inhabited this environment and surrounding areas would have been triggered. However, these changes have been poorly studied from a zooarchaeological perspective. The analysis of bone assemblages dated in historical times from lower Manso river valley (southwest Río Negro province) reveals the existence of continuities and changes in the interaction with animals. While the huemul (Hippocamelus bisulcus) maintains its central role in subsistence, established since the early Holocene, in historical times the contribution of complementary species is recorded in very low proportions. Among them is the horse (Equus caballus), whose remains suggest the 
consumption of meat and marrow in a similar way to that recorded among wild ungulates. The results show the importance of zooarchaeological studies at local scale and from a long term perspective.

KEY WORDS: ZOOARCHAEOLOGY, HISTORICAL TIMES, PATAGONIA, HUEMUL, HORSE

\section{INTRODUCCIÓN}

La llegada de los europeos a partir del siglo XVI no supuso la desarticulación completa de las sociedades preexistentes de la Patagonia argentina. Hasta fines del siglo XIX, mantuvieron una relativa autonomía política, social y económica cuando, a través de una serie de acciones militares conocidas como «Conquista del Desierto", el Estado argentino expandió sus fronteras internas avanzando sobre gran parte de esta región sudamericana (Briones \& Delrio, 2007). Dicha autonomía no debe entenderse como aislamiento, ya que los grupos que poblaban la Patagonia y que estaban fuera de la jurisdicción colonial o estatal, interactuaron con los europeos y criollos de manera directa o indirecta a lo largo de varios siglos, lo que llevó a la transformación de muchos aspectos de su organización (Ortelli, 1996; Nacuzzi, 1998; Bechis, 2004). La introducción y disponibilidad de nuevos animales, como caballos (Equus caballus), vacas (Bos taurus) y ovejas (Ovis aries), está entre algunos de los cambios más notables, los que fueron incorporados como alimento, medio de transporte, bien de prestigio y como un factor clave en un nuevo sistema económico basado en los rebaños capturados y/o criados al este de los Andes y comercializados del otro lado de la Cordillera (Mandrini, 1985; Nacuzzi, 2007).

En la estepa del centro-norte de Patagonia, estudios zooarqueológicos determinaron que algunos grupos continuaron basando la subsistencia en las mismas presas que durante milenios habían sido la base de su alimentación. Otros, sin abandonar la caza de las especies nativas, se volcaron a formas de producción que enfatizaron la cría y el intercambio de los nuevos animales (Silveira \& Cordero, 2014; Guillermo, 2017). Por ejemplo, en el sitio neuquino Casa de Piedra de Ortega, en los últimos ca. 200-280 años ${ }^{14} \mathrm{C}$ AP se registra el consumo alimenticio de caballo, vaca y, fundamentalmente, oveja aunque el guanaco (Lama guanicoe), el único ungulado nativo representado, sigue teniendo el papel dominante en la subsistencia (Guillermo, 2017). En cambio en el cercano sitio La Marcelina, aproximadamente para el mismo momento, el guanaco pierde importancia en relación con la crianza de ovejas, que deviene en la actividad principal (Silveira \& Cordero, 2014).

En el bosque andino, la presencia en contextos arqueológicos de restos óseos de fauna introducida fue explicada a partir de las fuentes documentales (Hajduk, 1991; Silveira, 1996; Hajduk \& Albornoz, 1999; Albornoz \& Hajduk, 2009; Hajduk et al., 2013). En estos trabajos se enfatiza en la información que menciona la existencia de fauna introducida en el área del lago Nahuel Huapi desde el siglo XVII (Florez de León, 1992), así como el pleno desarrollo de actividades pastoriles hacia fines del siglo XVIII, en esa zona y áreas circundantes (Fonck, 1896). Sin embargo, la carencia de estudios zooarqueológicos impide conocer los detalles de su aprovechamiento y el modo en que las nuevas especies se articularon con la forma de subsistencia preexistente. En el caso del bosque andino patagónico, la disponibilidad de nuevos animales puede haber sido de suma importancia, ya que este ambiente se caracteriza por una estructura de recursos faunísticos dominada por especies de pequeño tamaño corporal y el huemul (Hippocamelus bisulcus) el único ungulado de porte mediano-grande (Fernández, 2010). Además, se desconoce si los cambios se dieron del mismo modo en sectores con diferente grado de interacción entre indígenas y europeos y, por ende, con distinta frecuencia y tipo de acceso (directo, indirecto) a las especies introducidas.

Con el propósito de comprender cómo interactuaron las sociedades cazadoras-recolectoras del bosque andino del centro-norte de Patagonia con los animales durante los tiempos históricos, aquí se analiza el caso del valle del río Manso inferior. Está ubicado a $80 \mathrm{~km}$ al sur del área de Nahuel Huapi, en el suroeste de la provincia de Río Negro, Argentina (Figura 1) y posee una larga historia de ocupación humana que se inicia en el Holoceno temprano 


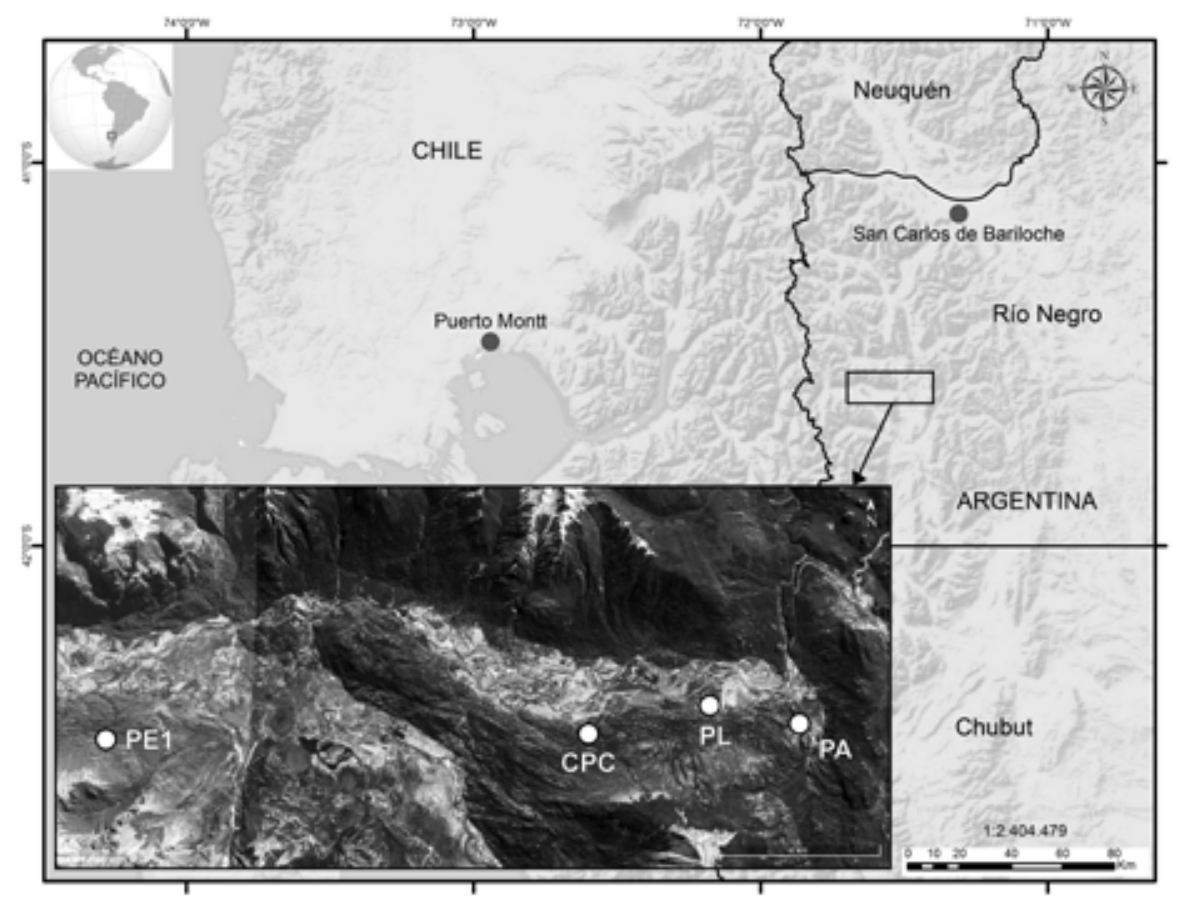

FIGURA 1

Esquema con la ubicación de los sitios del valle del Manso inferior mencionados en el texto.

(Bellelli et al., 2013; Fernández et al., 2013). Dentro del tramo final de ocupación del Manso inferior se identificó un lapso atribuido a tiempos históricos, para el que se toma como punto de inicio los años 1552 a 1567, cuando se produce la fundación de los asentamientos españoles de Valdivia, Villarica, Castro y Osorno. A partir de ese momento, comienza la integración del área de Nahuel Huapi a la esfera de influencia europea (Albornoz \& Montero, 2008), que pudo repercutir en las sociedades que ocupaban el Manso inferior. Esto se evidencia en la recuperación de algunos restos óseos de caballo (Fernández \& Carballido Calatayud, 2015) y en la presencia de dos motivos rupestres que originalmente representaban guanacos y que, con la adición de vasos y colas, fueron transformados en caballos (Podestá et al., 2005).

\section{EL MANSO INFERIOR: POBLAMIENTO E INTERACCIÓN CON LA FAUNA}

Las primeras ocupaciones del valle del río Manso inferior datan del Holoceno temprano ( $c a$. 8200-
7200 años ${ }^{14} \mathrm{C}$ AP) y se continúan en el Holoceno medio ( $c a .4700$ años ${ }^{14} \mathrm{C}$ AP). A principios del Holoceno tardío ( $c a .3500$ años ${ }^{14} \mathrm{C}$ AP) se vuelven más continuas y a partir de 1600 años ${ }^{14} \mathrm{C} \mathrm{AP}$, las sociedades que lo habitaron tuvieron una mayor permanencia y/o recurrencia en el uso de este sector del bosque (Bellelli et al., 2013; Fernández et al., 2013). En este último lapso hay un aumento de la señal arqueológica, los sitios poseen fechados continuos y superpuestos entre sí, se incrementa la frecuencia de artefactos y otras evidencias, comienza la actividad pictórica rupestre y las señales isotópicas de los restos humanos sugieren el consumo preferencial de recursos del bosque y el ecotono bosque-estepa (Bellelli et al., 2013; Fernández et al., 2013; Fernández \& Tessone, 2014; Fernández \& Carballido Calatayud, 2015).

A lo largo de toda la secuencia cronológica de ocupación del valle la presa principal fue el huemul, un cérvido de hasta $100 \mathrm{~kg}$ que conforma pequeños grupos sociales y se presenta espacialmente disperso (Vila et al., 2010). Este animal fue aprovechado por su carne y por la grasa contenida en la médula de los huesos de sus extremidades. En el Holoceno temprano y medio, el huemul aparece 
acompañado por presas de menor rinde económico como el zorro (Lycalopex sp.) y el pudú (Pudu puda), cuyos huesos también fueron aprovechados para confeccionar artefactos (Fernández, 2018). Durante el Holoceno tardío se siguen aprovechando estas mismas especies, con el agregado de puma (Puma concolor) y roedores Caviomorfos al consumo (Fernández \& Carballido Calatayud, 2015; Andrade \& Fernández, 2017).

\section{MATERIAL Y MÉTODOS}

Para este trabajo se consideraron como representativos de los tiempos históricos a los niveles de ocupación cuyas dataciones radiocarbónicas calibradas con dos sigmas incluyen en su distribución probabilística el rango 1552-1567 AD (Tabla 1). A partir de ello se conformaron las unidades de análisis PA0-5, que comprende los niveles 0 a 5 del sector sur de excavación del sitio Población Anticura; PLF19, correspondiente a la cuadrícula F19 del sitio Paredón Lanfré; PE1, que abarca los niveles 1 y 2 del sitio Peumayén 1 y CPC, que incluye la totalidad de lo excavado en el sitio Casa de Piedra Carro. Con el objetivo de explorar cambios y continuidades a través del tiempo, se incluyó en el análisis a la unidad PA6-9, conformada por los niveles 6 a 9 de Población Anticura, y datada entre 420 y 1410 AD (Fernández et al., 2013). Todos los sitios son aleros o reparos rocosos con manifestaciones

\begin{tabular}{|l|c|c|c|}
\hline Sitio-nivel & Código & $\begin{array}{c}\text { Fecha } \\
\text { (años A.P.) }\end{array}$ & Edad calibrada \\
\hline PE1-N2 & LP2161 & $210 \pm 60$ & 1645 AD: 1949 AD [100\%] \\
\hline PA-N3 & LP2413 & $280 \pm 40$ & $\begin{array}{c}\text { 1505 AD: } 1589 \text { AD [23\%] } \\
\text { 1617 AD: } 1681 \mathrm{AD}[46 \%] \\
\text { 1730 AD: } 1802 \mathrm{AD}[31 \%]\end{array}$ \\
\hline PA-N4 & LP2419 & $300 \pm 50$ & $\begin{array}{l}\text { 1485 AD: } 1678 \mathrm{AD}[83 \%] \\
\text { 1733 AD: } 1800 \mathrm{AD}[17 \%]\end{array}$ \\
\hline PLF19-N40-50 & LP1587 & $330 \pm 50$ & 1460 AD: $1670 \mathrm{AD}[99 \%]$ \\
\hline PA-N5 & LP2079 & $400 \pm 70$ & 1433 AD: $1653 \mathrm{AD}[100 \%]$ \\
\hline CPC-N10-20 & LP2143 & Moderno & - \\
\hline CPC-N20-30 & LP2158 & Moderno & - \\
\hline
\end{tabular}

Referencias: PA, Población Anticura, PL, Paredón Lanfré, PE1, Peumayén 1 y CPC, Casa de Piedra Carro. Fechas calibradas con el programa CALIB 7.0.4, de Stuiver y Reimer. 1993. Radiocarbon 35 (1): 215-230. Método de intersección (A).

TABLA 1

Fechados radiocarbónicos calibrados de los sitios del valle del Manso inferior cuya probabilidad incluye el rango 1552-1567 AD. Todas las fechas fueron obtenidas a partir de la datación de carbón vegetal. rupestres que fueron intervenidos con diferente intensidad. Como resultado, las unidades de análisis representan diferentes superficies y potencias. Por esta razón, para facilitar las comparaciones se normalizó la frecuencia de los materiales recuperados calculando su densidad por metro cúbico (Tabla 2).

\begin{tabular}{|l|r|r|r|}
\hline Unidad de análisis & \multicolumn{1}{|c|}{$\mathrm{M}^{3}$} & \multicolumn{1}{c|}{ NR } & NRnorm \\
\hline PA0-5 & 3 & 15002 & 5000,7 \\
\hline PA6-9 & 3 & 14632 & 4877,3 \\
\hline PLF19 & 0,5 & 12 & 24 \\
\hline CPC & 0,35 & 65 & 185,7 \\
\hline PE1 & 0,25 & 1 & 4 \\
\hline
\end{tabular}

TABLA 2

Volumen de sedimento excavado expresado en metros cúbicos $\left(\mathrm{M}^{3}\right)$, número de restos óseos recuperados (NR) y frecuencia de restos óseos normalizada por $\mathrm{m}^{3}$ en cada sitio (NRnorm).

Para estimar la abundancia taxonómica se consideraron el NISP y el MNI (Lyman, 1994). En los vertebrados de más de $5 \mathrm{~kg}$ se relevó la meteorización siguiendo los criterios de Behrensmeyer (1978). Las modificaciones de las superficies óseas se identificaron a ojo desnudo y con una lupa de bajos aumentos (10x) y comprenden los daños relacionados con la actividad de los carnívoros (Binford, 1981; Mondini, 2002), las improntas de raíces, las marcas de roedores y el pisoteo (Fiorillo, 1989; Lyman, 1994), las modificaciones químicas por manganeso (Shahack-Gross et al., 1997) y aquellas relacionadas con la actividad humana: huellas de corte, raspado y percusión, de las que se contemplaron su localización y frecuencia (Binford, 1981; Mengoni Goñalons, 1999). El análisis se completó con el relevamiento de la fragmentación y el registro de los huesos termoalterados según el color y el estado de la superficie (Shipman et al., 1984).

\section{RESULTADOS}

Los conjuntos de Población Anticura se distinguen por la gran cantidad de restos óseos que poseen (representan en conjunto el 99,7\% de la muestra analizada). Incluso aplicando la corrección por volumen de sedimento removido, superan con creces a los demás sitios (Tabla 2). Más allá de esta diferencia, en todos los conjuntos dominan los pequeños vertebrados, fundamentalmente los roedores 


\begin{tabular}{|c|c|c|c|c|c|c|c|c|c|c|c|c|c|c|c|}
\hline & \multicolumn{3}{|c|}{ PA0-5 } & \multicolumn{3}{|c|}{ PA6-9 } & \multicolumn{3}{|c|}{ PLF19 } & \multicolumn{3}{|c|}{$\mathrm{CPC}$} & \multicolumn{3}{|c|}{ PE1 } \\
\hline Taxa & NISP & $\%$ NISP & MNI & NISP & $\%$ NISP & MNI & NISP & $\%$ NISP & MNI & NISP & $\%$ NISP & MNI & NISP & $\%$ NISP & MNI \\
\hline Ave & 3 & 0,02 & 1 & 1 & 0,01 & 1 & 0 & 0 & 0 & 0 & 0,0 & 0 & 0 & 0,0 & 0 \\
\hline Peces & 2 & 0,02 & 1 & 0 & 0,0 & 0 & 0 & 0 & 0 & 0 & 0,0 & 0 & 0 & 0,0 & 0 \\
\hline Mammalia & 88 & 0,7 & 1 & 64 & 0,5 & 1 & 2 & 18,2 & 1 & 1 & 1,6 & 1 & 0 & 0,0 & 0 \\
\hline Artiodactyla & 31 & 0,2 & 1 & 18 & 0,14 & 1 & 1 & 9,1 & 1 & 0 & 0,0 & 0 & 0 & 0,0 & 0 \\
\hline L. guanicoe & 0 & 0,0 & 0 & 1 & 0,01 & 1 & 0 & 0 & 0 & 0 & 0,0 & 0 & 0 & 0,0 & 0 \\
\hline H. bisulcus & 354 & 2,8 & 5 & 123 & 0,95 & 3 & 3 & 27,3 & 1 & 0 & 0,0 & 0 & 1 & 100,0 & 1 \\
\hline P. puda & 21 & 0,2 & 1 & 4 & 0,03 & 1 & 0 & 0 & 0 & 2 & 3,2 & 1 & 0 & 0,0 & 0 \\
\hline E. caballus & 6 & 0,05 & 1 & 0 & 0,0 & 0 & 0 & 0 & 0 & 0 & 0,0 & 0 & 0 & 0,0 & 0 \\
\hline B. taurus & 3 & 0,02 & 1 & 0 & 0,0 & 0 & 0 & 0 & 0 & 0 & 0,0 & 0 & 0 & 0,0 & $\underline{0}$ \\
\hline P. concolor & 9 & 0,1 & 1 & 0 & 0,0 & 0 & 0 & 0 & 0 & 0 & 0,0 & 0 & 0 & 0,0 & $\underline{0}$ \\
\hline Lycalopex sp. & 19 & 0,1 & 1 & 12 & 0,09 & 1 & 0 & 0 & 0 & 0 & 0,0 & 0 & 0 & 0,0 & 0 \\
\hline Dasipodidae & 10 & 0,09 & 1 & 2 & 0,02 & 1 & 0 & 0 & 0 & 0 & 0,0 & 0 & 0 & 0,0 & 0 \\
\hline Rodentia & 12259 & 95,7 & $*$ & 12695 & 98,25 & $*$ & 5 & 45,4 & 4 & 60 & 95,2 & 3 & 0 & 0,0 & 0 \\
\hline Total & 12805 & 100,0 & 17 & 12920 & 100,0 & 11 & 11 & 100 & 7 & 63 & 100,0 & 5 & 1 & 100,0 & 1 \\
\hline
\end{tabular}

TABLA 3

Composición taxonómica de los conjuntos óseos expresada en NISP, \%NISP y MNI. En el caso de los roedores esta última medida está estimada sobre cráneos y mandíbulas. El símbolo * significa que el MNI no ha sido calculado.

(Tabla 3). El análisis de estos últimos está en curso, y hasta el momento pudo determinarse que una buena parte proviene de la disgregación de egagrópilas y/o de la descomposición de heces de mamíferos carnívoros. Dejando de lado los restos de pequeños vertebrados, las unidades de análisis PLF19, CPC, PE1 presentan seis, tres y un espécimen, respectivamente (Tabla 3). En PLF19 una epífisis distal de primera falange y un fragmento de epífisis distal de húmero fueron atribuidos a Mammalia y un pequeño fragmento de diente fue asignado a Artiodactyla. El huemul está representado por una bula timpánica, una epífisis proximal de primera falange y una diáfisis proximal de fémur, la cual posee huellas de corte y percusión. En CPC hay una astilla de hueso largo clasificada como Mammalia y una diáfisis de metacarpo y una epífisis distal de metapodio, ambas de pudú. La única modificación observada son las improntas de raíces sobre el hueso de Mammalia. El único resto de PE1 corresponde a un fragmento de pelvis de Mammalia.

En las dos unidades de análisis de Población Anticura (PA0-5 y PA6-9) dominan los restos de huemul, seguidos por dos categorías taxonómicas de menor resolución (Mammalia y Artiodactyla), que representan entre $22 \%$ y $32 \%$ de los restos (Figura 2). Además, hay bajas proporciones de aves, zorro, pudú y armadillo (Dasipodidae). Exclusivos de la unidad PA0-5, correspondiente a los tiempos históricos, se registran restos de peces, puma, caballo y vaca (Figura 3). Un solo espécimen de guanaco, ungulado que actualmente no habita el bosque en esta zona de Patagonia, fue recuperado en la unidad PA6-9.

Las modificaciones de las superficies óseas sugieren la acción de varios actores y procesos tafonómicos de origen natural. En las unidades de pocos especímenes se registraron marcas de raíces en un hueso de Mammalia de CPC y marcas de roedor en espécimen de huemul de PLF19. En todos los taxones y en ambas unidades de PA las modificaciones más frecuentes son las de carnívoros. La presencia de materiales con evidencias de corrosión digestiva sugiere que parte de los conjuntos son de origen escatológico. Destaca en la unidad PA0-5 que la totalidad de los restos de aves (NISP 3), 37\% de zorro y 30\% de los especímenes de pudú exhiben este tipo de modificación mientras que en PA6-9 la única incidencia elevada de daños por digestión se observa en pudú ( $25 \%$ del NISP). La gran proporción de huesos mascados y digeridos que se observa en los niveles más recientes excede a la registrada en observaciones tafonómicas efectuadas en una madriguera de zorro del mismo ambiente (Fernández et al., 2010). Este hecho, junto con la morfología de los daños (ver Fernández \& Carballido Calatayud, 2015: figura 5), nos lleva a proponer como hipótesis a evaluar en trabajos futuros, que las modificaciones observadas podrían ser producto de la acción de perros. Éstos habrían actuado de manera coetánea a la ocupación humana del sitio o bien con posterioridad, en ocasión del uso reciente del lugar. Entre las otras modificaciones registradas en PA, las marcas de roedor se 


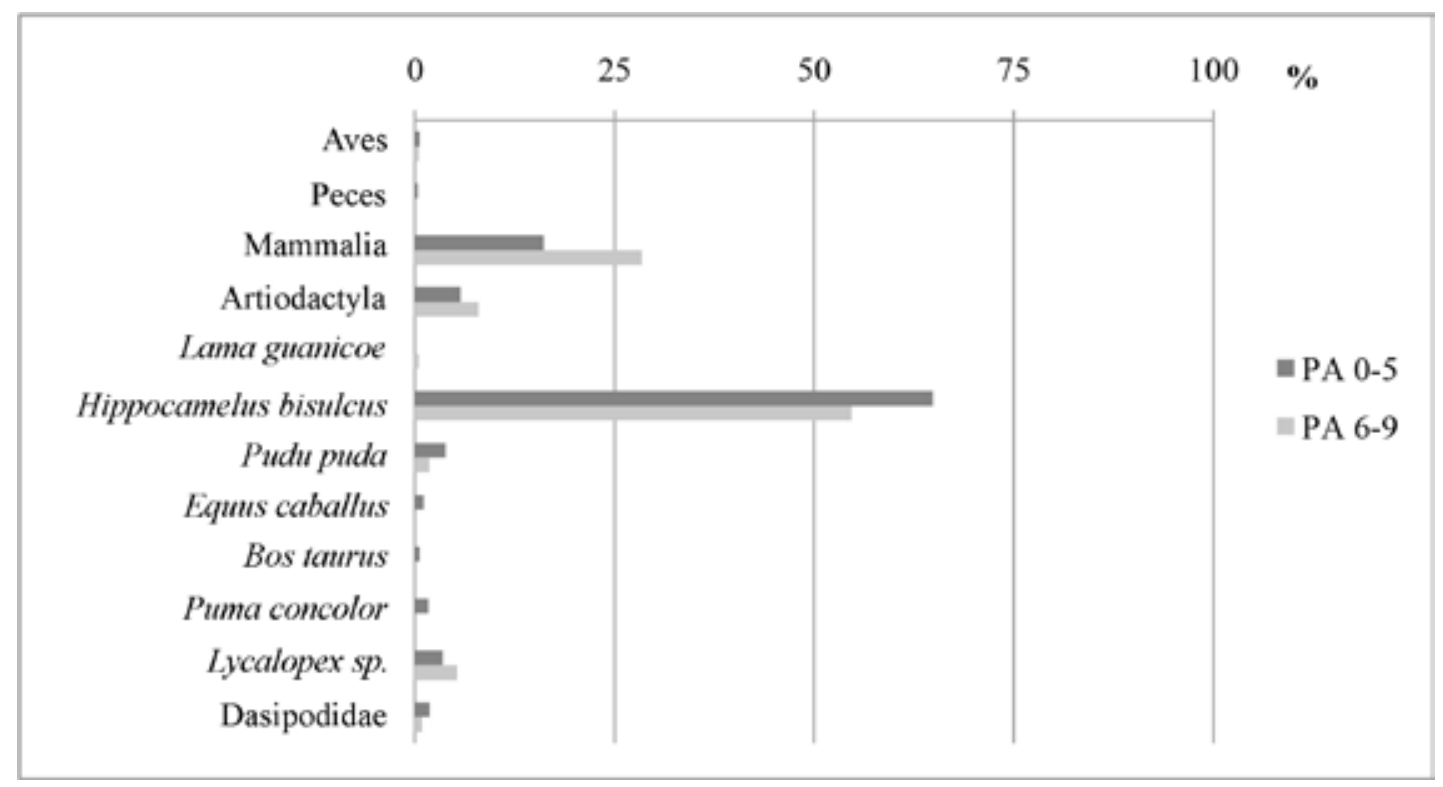

FIGURA 2

Abundancia taxonómica relativa (excluyendo roedores) registrada en las dos unidades del sitio PA.

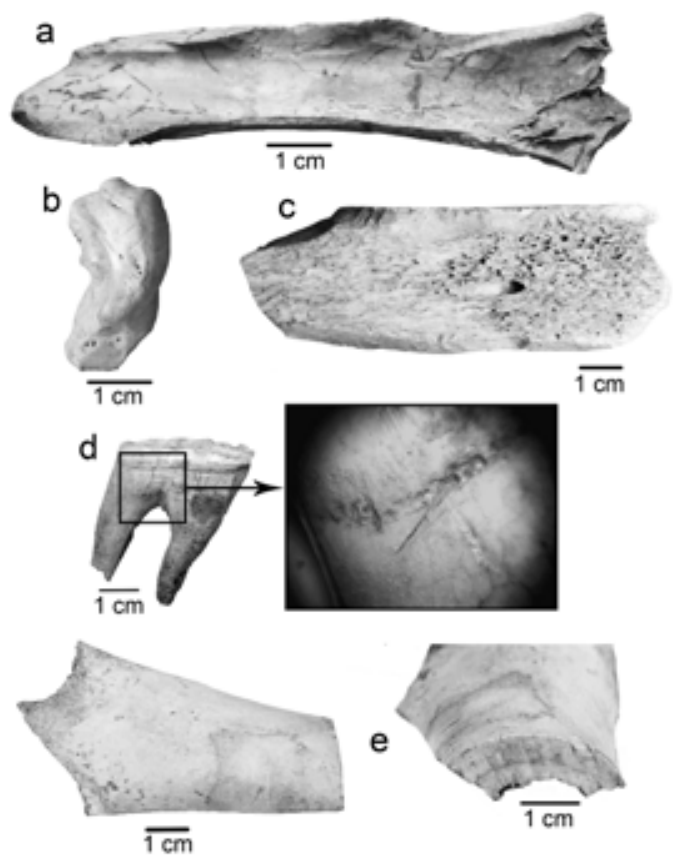

FIGURA 3

Restos óseos de la unidad PA0-5 con evidencias de procesamiento. a: fémur de huemul con negativos de impacto internos; b: escafolunar del puma con huellas de corte; c: tibia de caballo con negativos de impacto internos; d: DP2 deciduo de caballo, con huellas de corte (detalle) y e: fémur de vaca con corte de sierra circular. presentan en $15 \%$ a $20 \%$ de los taxones con NISP más alto. Las marcas de pisoteo y raíces afectan a una baja proporción de huesos $(<4 \%)$ de algunos taxones mientras que las evidencias de tinción por manganeso son más importantes en la unidad PA6-9, lo cual podría estar relacionado con que los restos estuvieron sepultados más tiempo (Tabla 4).

Las evidencias de procesamiento se observaron en ocho de los diez taxones identificados en la unidad PA0-5: huemul, pudú, zorro, puma, caballo y vaca (Figura 3) así como en las categorías de grano grueso Artiodactyla y Mammalia (Tabla 4). En la unidad PA6-9 están presentes en estas dos últimas categorías así como en huemul y guanaco (Tabla 4). Las huellas de procesamiento en los restos de huemul y de pudú sugieren el aprovechamiento de la carne y la médula de estos animales. En cambio, las que están presentes en un sólo espécimen de zorro - un fragmento de atlas con huellas de corte- son difíciles de relacionar con un tipo de aprovechamiento específico ya que la separación de la cabeza pudo darse tanto en el marco de un procesamiento con fines alimenticios como para obtener la piel. Por otra parte, la elevada proporción de especímenes de zorro con marcas de carnívoros $(73 \%)$ sugiere que la acción humana tuvo un rol menor en la conformación de este conjunto de PA0-5. El único espécimen de guanaco, un fragmento de metapodio distal recuperado en unidad PA6-9, posee 


\begin{tabular}{|l|r|r|r|r|r|r|r|r|r|r|r|r|}
\hline Unidad & \multicolumn{1}{|c|}{ PA0-5 } & \multicolumn{1}{c|}{ PA6-9 } \\
\hline Modificación & $\mathrm{H}$ & \multicolumn{1}{c}{$\mathrm{C}$} & $\mathrm{R}$ & $\mathrm{P}$ & $\mathrm{Ra}$ & $\mathrm{Mn}$ & $\mathrm{H}$ & $\mathrm{C}$ & $\mathrm{R}$ & $\mathrm{P}$ & $\mathrm{Ra}$ & \multicolumn{1}{c|}{ Mn } \\
\hline Ave & 0 & 100 & 0 & 0 & 0 & 0 & 0 & 0 & 0 & 0 & 0 & 0 \\
\hline Mammalia & 21,6 & 46,6 & 23,9 & 2,3 & 2,3 & 8 & 29,7 & 29,7 & 14,1 & 0 & 9,4 & 17,2 \\
\hline Artiodactyla & 22,6 & 35,5 & 19,4 & 0 & 3,2 & 0 & 44,1 & 5,6 & 0 & 0 & 0 & 0 \\
\hline H. bisulcus & 38,7 & 43,5 & 18,4 & 1,1 & 5,4 & 2,3 & 32,5 & 23,6 & 19,5 & 3,3 & 14,6 & 10,6 \\
\hline P. puda & 9,5 & 33,3 & 0 & 0 & 4,8 & 0 & 0 & 25 & 0 & 0 & 0 & 0 \\
\hline Lycalopex sp. & 5,2 & 73,3 & 5,3 & 0 & 0 & 5,3 & 0 & 8,3 & 25 & 0 & 0 & 0 \\
\hline P. concolor & 55,5 & 44,4 & 33,3 & 0 & 0 & 0 & - & - & - & - & - & - \\
\hline E. caballus & 50 & 33,3 & 50 & 0 & 16,7 & 16,7 & - & - & - & - & - & - \\
\hline B. taurus & 66,7 & 100 & 66,7 & 0 & 0 & 0 & - & - & - & - & - & - \\
\hline
\end{tabular}

TABLA 4

Proporción de especímenes con modificaciones óseas en PA. H: huellas de procesamiento; C: marcas de carnívoros; R: marcas de roedores; P: pisoteo; Ra: marcas de raíces; Mn: manchas de manganeso.

huellas alrededor del cóndilo similares a las dejadas cuando se busca realizar la fractura controlada de una pieza ósea. Se considera que fue transportada adherida al cuero de un animal cazado en la estepa o en el ecotono bosque-estepa (Fernández \& Carballido Calatayud, 2015). Las huellas de corte en pelvis, costillas, vértebras y huesos largos de puma sugieren un uso alimenticio. Si bien no se encontraron evidencias de cuereo, no descartamos el aprovechamiento complementario de la piel.

Entre las especies introducidas, los dientes de caballo presentan huellas de corte que podrían estar ligadas a la extracción del cuero. Además, un fragmento de tibia posee huellas de corte y percusión que indican el consumo de carne y médula, esto último en lo que podríamos denominar «un modo tradicional", esto es, similar al registrado en ungulados silvestres. En cambio, uno de los restos de vacuno presenta un corte cuya morfología corresponde a una sierra eléctrica. Sumado a su posición estratigráfica, sugieren que se trata de restos asociados con ocupaciones recientes del alero. Los especímenes de peces corresponden a dos vértebras de pequeño tamaño, una de ellas quemada. La ausencia de evidencias de corrosión digestiva, la distancia del sitio al río Manso y presencia de termoalteración llevan a considerarlas como restos consumidos por los cazadores-recolectores.

\section{DISCUSIÓN}

El estudio de los cuatro conjuntos asignados a los tiempos históricos y su comparación con PA6-9 muestra que, a pesar de la disponibilidad de nuevas especies, el huemul continuó dominando la subsis- tencia y que las especies exóticas no aportaron sustantivamente al consumo. El caballo fue aprovechado de manera complementaria junto con otras especies -pudú, zorro, puma y peces- y sólo está presente en la unidad PA0-5. La falta de peso de las especies introducidas no puede explicarse por su escasez o ausencia, ya que estaban disponibles en zonas relativamente cercanas desde el siglo XVII (Florez de León, 1992). Estos animales, además de estar en poder de los indígenas, se asilvestraron y se transformaron en un nuevo recurso. El relato del explorador chileno Hans Steffen, que recorrió el río Manso en 1896, es elocuente al respecto. Comenta la caza una vaca salvaje (bagual) que formaba parte de un grupo de 200 animales y señala que dentro del bosque, el viajero siempre contará con carne para sus travesías gracias a la existencia de estos baguales (Steffen, 1909-1910: 164). Destaca entonces, que la disponibilidad de dos nuevos ungulados no haya modificado la subsistencia durante los tiempos históricos. Una explicación alternativa sobre la falta de evidencias del aprovechamiento de animales exóticos es que fueron faenados en otros lugares, diferentes a aleros y reparos rocosos examinados en este trabajo. El registro arqueológico disponible en el Manso inferior no permite avanzar en la resolución de esta hipótesis. No obstante, de haber existido una segmentación espacial en el manejo de las especies introducidas, sería esperable encontrar sus restos entre los desechos de alimentación, tal como ocurre en los aleros mencionados en la introducción, que al igual que los tratados aquí, funcionaron como loci de consumo (Silveira \& Cordero, 2014; Guillermo, 2017).

Incluso al considerar las variaciones relacionadas con la historia de ocupación de los sitios del valle del río Manso, sus condiciones de preservación 
y el tamaño de la superficie excavada en cada uno de ellos, el huemul es la especie más representada en términos de NISP y la que exhibe una mayor proporción de huellas relacionadas con el aprovechamiento humano. Hasta el momento, la explotación del huemul era entendida como resultado de su abundancia natural y la disponibilidad y escasez de las presas alternativas (Fernández et al., 2016). El caso que presentamos sugiere que también se dieron situaciones en que se prefirió este cérvido por sobre otras especies, que quizá fueron valoradas diferencialmente, como resulta evidente en el caso del caballo, cuyo rol principal fue el de medio de transporte y bien de prestigio (Nacuzzi, 2007).

Tal como se refiere en el título del trabajo, los resultados del análisis muestran continuidad y cambios en el modo de interactuar con la fauna en este sector del bosque de Patagonia. Es posible que la primera esté vinculada con elecciones sobre qué animales privilegiar para el consumo alimenticio mientras que los cambios derivados de la introducción de las nuevas especies pudieron darse en el marco de una mayor diversidad de recursos faunísticos aprovechados, cuyas causas aún están siendo evaluadas. Sin embargo, se observa que el caballo fue incorporado a la esfera de subsistencia de las poblaciones indígenas manteniendo el "modo tradicional" de aprovechamiento, lo cual también da cuenta de una continuidad dentro de los cambios acaecidos. Finalmente, es probable que la adopción de las nuevas especies tuviera otros fines que no han quedado reflejados en los contextos analizados.

\section{CONCLUSIONES}

Usualmente, el hallazgo de restos óseos de especies introducidas en contextos de cazadores-recolectores fue interpretado como un indicador cronológico y como evidencia de contacto indígena-europeo (Silveira, 1996; Hajduk et al., 2013). Alternativamente, y junto con otros indicios, estos restos también se utilizaron para discutir los procesos de formación del registro (Andrade \& Fernández, 2017), incluyendo el uso actual y/o relativamente reciente de los sitios (Silveira, 1999).

La identificación de los tiempos históricos como problema de estudio en contextos sin restos materiales "de contacto" o con pocos indicadores constituye un aporte para la arqueología del bosque andino del centro-norte de Patagonia. Su defi- nición implica enfrentar los desafíos derivados de la baja resolución temporal que usualmente presentan estas ocupaciones, lo que obliga a destinar recursos para fechar y caracterizar los primeros niveles de los depósitos arqueológicos, frecuentemente relegados por considerárselos palimpsestos y/o contaminados por ocupaciones recientes. Desde la perspectiva zooarqueológica, esto implica que la evaluación tafonómica y de los procesos de formación del registro son aspectos indispensables para el análisis de este lapso. Asimismo, la mera presencia de restos óseos de especies exóticas no da cuenta por sí misma del modo en que fueron aprovechadas y mucho menos, de la existencia de cambios en la subsistencia. Los casos zooarqueológicos conocidos -a los que ahora sumamos los del Manso inferior- sugieren que, si se quieren explicar los procesos de cambio social relacionados con la incorporación de nuevos animales, el registro documental no sólo debe ser complementado sino también interrogado/impugnado desde el registro arqueológico (Goñi, 2000; Goñi \& Nuevo Delaunay, 2009). Más aún, en áreas con pocos o nulos registros escritos y/o alejadas de los principales centros de interacción entre indígenas y europeos.

En el valle del río Manso inferior, el registro zooarqueológico muestra que dentro de la gama de interacciones posibles entre las sociedades indígenas y las especies introducidas, la subsistencia se mantuvo centrada en el huemul, como en los milenios anteriores. Si bien numerosos factores pudieron influir en el mantenimiento de las formas tradicionales de subsistencia, uno que se revela de forma sugerente es que se trata posiblemente de una decisión de los grupos humanos que ocuparon el valle. Esto queda, de alguna manera, reflejado en la incorporación del caballo de forma complementaria al aprovechamiento de las especies nativas. Más estudios desde esta nueva mirada, preferentemente a escala regional, permitirán dar cuenta de la variedad de situaciones posibles a partir de la disponibilidad de los nuevos animales.

\section{AGRADECIMIENTOS}

Agradecemos a Ana Forlano por la composición de las imágenes y al Área Geomática del INAPL por la confección del mapa de la Figura 1. Nuestra gratitud con los pobladores del Manso inferior así como 
con el resto del equipo de investigación arqueológica del área. Agradecemos también los comentarios y sugerencias de los dos evaluadores anónimos que contribuyeron a mejorar el manuscrito. Los trabajos fueron financiados por la Agencia Nacional de Promoción Científica y Tecnológica (PICT 049976; 06-26332 y 2012-0471), el Consejo Nacional de Investigaciones Científicas y Tecnológicas (PIP 119/1998; 232/2010; 365/2014), la Universidad de Buenos Aires (UBACyT: U603/2001-2003) y el INAPL (Ministerio de Cultura de la Nación).

\section{REFERENCIAS}

Albornoz, A. \& Hajduk, A. 2009: «Ladran Sancho I»Jinetes y caballos en el arte rupestre en la arqueología y la etnohistoria del área del Nahuel Huapi. Trabajo presentado a las XII Jornadas Interescuelas, Departamentos de Historia. Bariloche. Ms.

Albornoz, A.M. \& Montero, G. 2008: Nahuel Huapi: antropología e historia regional de un área de frontera. En: CD Rom de las III Jornadas de Historia de la Patagonia, San Carlos de Bariloche, 6-8 de noviembre de 2008.

Andrade, A. \& Fernández, P.M. 2017: Rodent consumption by hunter-gatherers in North Patagonian Andean forests (Argentina): insights from the small vertebrate taphonomic analysis of two Late Holocene archaeological sites. Journal of Archaeological Science: Reports 11: 390-399.

BECHIS, M. 2004: Cacicazgos pampeanos: fronteras adentro, fronteras afuera. Revista TEFROS 6(1).

Behrensmeyer, A. 1978: Taphonomic and ecologic information from bone weathering. Paleobiology 4: 130162.

Bellelli, C.; Carballido Calatayud, M. \& Fernández, P.M. 2013: Ocupaciones tempranas del bosque norpatagónico: El sitio Población Anticura, Valle del Río Manso Inferior, Río Negro. En: Bárcena, R. \& Martín, S.E. (eds.): Arqueología Argentina en el Bicentenario de la Asamblea General Constituyente de 1813: 516. La Rioja, Universidad Nacional de La Rioja.

BINFORD, L. 1981: Bones. Ancient Men and Modern Myths. Academic Press, New York.

Briones, C. \& Delrio, W. 2007: La «Conquista del Desierto» desde perspectivas hegemónicas y subalternas. Runa XXVII: 23-48.

Caracotche, S.; Margutti, L. \& Cabrera, S. 2010: Memorias para las historias de El Manso. Administración de Parques Nacionales, S.C. de Bariloche.
FernáNDEZ, M.G. 2018: El aprovechamiento de la fauna en el interior del bosque durante el Holoceno temprano y medio. El caso del sitio Población Anticura (provincia de Río Negro, Argentina). La Zaranda de Ideas. (En prensa).

FernáNDEZ, P.M. 2010: Cazadores y presas. 3500 años de interacción entre seres humanos y animales en el noroeste de Chubut. Fundación de Historia Natural Félix de Azara, Buenos Aires.

Fernández, P.M. \& Carballido Calatayud, M. 2015: Armas y presas. Técnicas de caza en el interior del bosque patagónico. Relaciones de la Sociedad Argentina de Antropología XL (1): 279-301.

Fernández, P.M. \& Tessone, A. 2014: Modos de ocupación del bosque patagónico de la vertiente oriental de los andes: aportes desde la ecología isotópica. Revista Chilena de Antropología 30: 83-89.

Fernández, P.M.; Cruz, I. \& Forlano, A. 2010: Sitio 37: una madriguera de carnívoro en el norte de la Patagonia Andina (Cholila, Provincia de Chubut, Argentina). En: Gutiérrez, M.A.; De Nigris, M.; Fernández, P.M.; Giardina, M.; Gil, A.F.; Izeta, A.; Neme, G. \& Yacobaccio, H.D. (eds.): Zooarqueología a principios del siglo XXI: aportes teóricos, metodológicos y casos de estudio: 243-257. Ediciones del Espinillo, Buenos Aires.

Fernández, P.M.; Carballido Calatayud, M.; Bellelli, C. \& Podestá, M. 2013: Tiempo de cazadores. Cronología de las ocupaciones humanas en el valle del río Manso inferior (Río Negro). En: Zangrando, A.F.; Barberena, R.; Gil, A.; Neme, G.; Giardina, M.; Luna, L.; Otaola, C.; Paulides, S.; Salgán, L. \& Tivoli, A. (eds.): Tendencias teórico-metodológicas y casos de estudio en la arqueología de Patagonia: 167-175. Museo de Historia Natural de San Rafael, Sociedad Argentina de Antropología e Instituto Nacional de Antropología y Pensamiento Latinoamericano, Buenos Aires.

Fernández, P.M.; Cruz, I.; Belardi, J.B.; De Nigris, M. \& MuÑoz, S. 2016: La explotación del huemul (Hippocamelus bisulcus, Molina 1782) en la Patagonia a lo largo del Holoceno. Magallania 44(1): 187-209.

FioRILlo, A. 1989: An Experimental Study of Trampling: Implications for the Fossil Record. In: Bonnischen, R. \& Sorg, M.H. (eds.): Bone Modification: 61-72. Orono, Maine, Centre for the Study of the First Americans, Institute for Quaternary Studies, University of Maine.

Florez de León, D. 1992: Florez de León, Diego «Memorial”. Publicación del Museo de la Patagonia F. P. Moreno, Bariloche.

Fonck, F. 1896: Viajes de Fray Francisco Menéndez. a Nahuel Huapi; publicados y comentados por F. Fonck, Valparaíso, Edición Centenaria. 
GoÑI, R. 2000: Arqueología de momentos históricos fuera de los centros de conquista y colonización: un análisis de caso en el sur de la Patagonia. En: Belardi, J.B.; Carballo Marina, F. \& Espinosa, S. (eds.): Desde el País de los Gigantes. Perspectivas arqueológicas en Patagonia: 283-296. UNPA, Río Gallegos.

Goñi, R. \& Nuevo Delaunay, A. 2009: La Arqueología como «fuente»de la Historia. En: Salemme, M.; Santiago, F.; Álvarez, M.; Piana, E.; Vázquez, M. \& Mansur, E. (eds.): Arqueología de la Patagonia. Una mirada desde el último confín: 149-158. Ushuaia, Utopías.

Guillermo, A. 2017: Zooarqueología de la transición prehispánica y posthispánica del sitio arqueológico Casa de Piedra de Ortega (Río Negro, Argentina). Tesis de Licenciatura en Ciencias Antropológicas, Facultad de Filosofía y Letras, Universidad de Buenos Aires. Ms.

HAJDuK, A. 1991: Sitio arqueológico hispano-indígena, localizado en el actual predio del Club deportivo Llao Llao. Depto Bariloche. Pcia. Río. Negro. Comunicaciones Científicas del Museo de la Patagonia F. P. Moreno, Serie Antropología, Año 2, No 2, San Carlos de Bariloche.

Hajduk, A. \& Albornoz, A.M. 1999: El sitio Valle Encantado I. Su vinculación con otros sitios. Un esbozo de la problemática local diversa del Nahuel Huapi. En: Soplando en el Viento... Actas de las Terceras Jornadas de Arqueología de la Patagonia: 371-391. INAPL-Universidad del Comahue, Neuquén-Buenos Aires.

Hajduk, A.; Albornoz, A.m.; Lezcano, M.J. \& Montero, G. 2013: De Chiloé al Nahuel Huapi. Nuevas evidencias materiales del accionar jesuítico en el gran lago (siglos XVII y XVIII). En: Nicoletti, M.A. \& Núñez, P. (comps.): Araucanía-Norpatagonia: la territorialidad en debate. Perspectivas ambientales, culturales, sociales, políticas y económicas: 243-279. IIDyPCa, Universidad Nacional de Río Negro, San Carlos de Bariloche.

Lyman, R. L. 1994: Vertebrate Taphonomy. Cambridge University Press, Cambridge.

MANDRINI, R. 1985: La sociedad indígena de las pampas en el siglo XIX. En: Lischetti, M. (comp.): Antropología: 205-230. EUDEBA, Buenos Aires.

Mengoni Goñalons, G.L. 1999: Cazadores de guanacos de la estepa patagónica. Colección Tesis Doctorales, Sociedad Argentina de Antropología, Buenos Aires.

MondinI, M. 2002: Formación del registro arqueofaunístico en abrigos rocosos de la Puna argentina. Ta- fonomía de carnívoros. Tesis de Doctorado, Facultad de Filosofía y Letras, Universidad de Buenos Aires, Buenos Aires.

NAcuzzI, L. 1998: Identidades Impuestas. Tehuelches, Aucas y Pampas en el Norte de la Patagonia. Sociedad Argentina de Antropología, Buenos Aires.

- 2007: Los grupos nómades de la Patagonia y el Chaco en el siglo XVIII: identidades, espacios, movimientos y recursos económicos ante la situación de contacto. Una reflexión comparativa. Chungara, Revista de Antropología Chilena 39(2): 221-234.

ORTELLI, S. 1996: La «araucanización» de las pampas: ¿realidad histórica o construcción de los etnólogos? Anuario del IEHS 11: 203-225.

Podestá, M.m.; Paunero, R. \& Rolandi, D. 2005: El Arte Rupestre de Argentina Indígena. Patagonia. Academia Nacional de la Historia y Union Academique International, Buenos Aires.

Shahack-Gross, R.; Bar-Yosef, O. \& Weiner, S. 1997: Black-coloured bones in Hayonim Cave, Israel: differentiating between burning and oxide staining. Journal of Archaeological Science 24: 439-446.

Shipman, P.; Foster, G. \& Schoeninger, M. 1984: Burnt Bones and Teeth: an Experimental Study of Color, Morphology, Crystal Structure and Shrinkage. Journal of Archaeological Science 11: 307-325.

Silveira, M. 1996: Alero Los Cipreses. En: Gómez Otero, J. (ed.): Arqueología. Solo Patagonia. Ponencia de las Segundas Jornadas de Arqueología de la Patagonia: 107-118. CENPAT-CONICET, Puerto Madryn.

- 1999: Alero Cicuta (Departamento Los Lagos, Provincia del Neuquén, Argentina). En: Soplando en el Viento. Actas de las Terceras Jornadas de Arqueología de la Patagonia: 561-576. INAPL-Universidad Nacional del Comahue, Neuquén-Buenos Aires.

Silveira, M. \& Cordero, A. 2014: Zooarqueología del sitio La Marcelina 1 (Provincia de Río Negro, Argentina). Atek Na 4: 67-141.

Stefren, H. 1909-1910: Viajes de exploración y estudio en la Patagonia occidental (1892-1902). Imprenta Cervantes, Santiago de Chile.

Vila, A.R.; Saucedo Galvez, C.E.; AldRidge, D.; Ramilo, E. \& CoRtr González, P. 2010: South Andean Huemul Hippocamelus bisulcus (Molina 1782). In: Duarte, J.M.B \& González, S. (eds.): Neotropical cervidology: Biology and Medicine of Latin American Deer: 89-100. Jaboticabal, Funep y Gland y IUCN. Brasil y Suiza. 